\title{
Understanding How an Invasive Vector Drives Pierce's Disease Epidemics: Seasonality and Vine-to-Vine Spread
}

\author{
Matthew P. Daugherty† and Rodrigo P. P. Almeida
}

Department of Entomology, University of California, Riverside 92521 and Department of Environmental Science, Policy and Management, University of California, Berkeley 94720.

Accepted for publication 15 November 2018.

\begin{abstract}
For vector-borne plant pathogens, disease epidemics may be attributable to multiple mechanisms, including introduction of a novel vector whose epidemiological role differs from that of native vectors. In such cases, understanding an exotic vector's ability to drive an epidemic is central to mitigating its impact. We studied how the invasive glassywinged sharpshooter (Homalodisca vitripennis Germar) can drive Pierce's disease outbreaks in vineyards, focusing on its potential to promote vine-to-vine (i.e., secondary) spread of Xylella fastidiosa relative to potential constraints stemming from seasonality in the pathosystem. First, we developed a general vector-borne disease model to understand the consequences for disease dynamics of (i) seasonal acquisition efficiency and (ii) seasonal host recovery from infection. Results of the modeling indicate that these two sources of seasonality could constrain disease incidence, particularly when working in concert. Next, we

established a field cage experiment to determine whether $H$. vitripennis promotes vine-to-vine spread, and looked for evidence of seasonality in spread. Broadly, results from the experiment supported assumptions of the model; there was modest to significant increase in the frequency of pathogen spread over the first season, and those new infections that occurred later in the season were more likely to recover during winter. Ultimately, by the end of the second season, there was not evidence of significant secondary spread, likely due to a combination of seasonal constraints and low transmission efficiency by $H$. vitripennis. Collectively, these results suggest that, although $H$. vitripennis may be able to promote vine-to-vine spread in certain contexts, it may not be the key factor explaining its impact. Rather, the ability of $H$. vitripennis to drive epidemics is likely to be more directly related to its potential to reach higher population densities than native vectors.
\end{abstract}

For vector-borne pathosystems, disease epidemics may be attributable to a wide range of factors, including introduction of a novel pathogen, climatic conditions or climate change, landscape context or land-use change, or introduction of a novel vector species (Anderson et al. 2004; Sicard et al. 2018). Identifying which mechanism drives an epidemic is important for mitigating its impact but may be challenging for pathosystems in which multiple drivers are relevant. The plant pathogen Xylella fastidiosa exemplifies the potential for multiple ecological or evolutionary triggers of disease epidemics (Sicard et al. 2018). Outbreaks of X. fastidiosa (Wells et al. 1987) diseases have been attributed to pathogen introduction (Saponari et al. 2013), alternative hosts (Hewitt and Houston 1941), and invasion by a new vector (Hopkins and Purcell 2002). Here, we focus on the last of these, investigating the mechanism by which an invasive vector drives outbreaks of Pierce's disease (PD) in California vineyards (Almeida et al. 2005a).

$X$. fastidiosa can infect a broad range of agricultural, ornamental, and native plants (Hopkins and Purcell 2002; Purcell 1997). Among the most susceptible hosts is Vitis vinifera L., in which $X$. fastidiosa subsp. fastidiosa causes PD (Davis et al. 1978). Since first being reported in Southern California in the late 1880s (Pierce 1892), PD has affected grape production throughout the region, punctuated by a handful of severe epidemics. One of the most severe epidemics started circa 2000 in Southern California and the southern San

†Corresponding author: Matthew P. Daugherty; E-mail: matt.daugherty@ucr.edu

Funding: This research was supported by grants from the Consolidated Central Valley Table Grape Pest and Disease Control District to R. P. P. Almeida and M. P. Daugherty and the Pierce's Disease Control Program to R. P. P. Almeida.

*The $\boldsymbol{e}$-Xtra logo stands for "electronic extra" and indicates that six supplementary figures and three supplementary appendices are published online.

(C) 2019 The American Phytopathological Society
Joaquin Valley following the invasion of the glassy-winged sharpshooter, Homalodisca vitripennis (Germar, 1821) (Hemiptera: Cicadellidae) (Almeida et al. 2005a; Blua et al. 1999; Germar 1821; Perring et al. 2001).

$X$. fastidiosa is transmitted by insects that feed on xylem sap (Redak et al. 2004), which includes sharpshooter leafhoppers (Hemiptera: Cicadellidae, Cicadellinae) and spittlebugs (Hemiptera: Cercopoidea). There is no vertical or transtadial transmission of $X$. fastidiosa in these vectors but, once the pathogen is acquired, there is little to no latent period and adult insects maintain the infections in a persistent manner (Almeida and Purcell 2003; Freitag 1951; Purcell and Finlay 1979; Severin 1949). The most efficient vector of $X$. fastidiosa to grapevines in California is the blue-green sharpshooter, Graphocephala atropunctata Signoret (Daugherty and Almeida 2009; Severin 1949), whose impact in coastal vineyards has been well characterized (Hewitt et al. 1942; Purcell 1974, 1975). Yet, for other grape-growing areas, there are other sharpshooter species that play a more important role in PD epidemics, such as $H$. vitripennis in the southern part of the state (Almeida et al. 2005a).

H. vitripennis was introduced into Southern California by 1990 (Sorensen and Gill 1996). Over the next decade, it spread throughout Southern California and into parts of the San Joaquin Valley, ultimately reaching high population densities; by the late 1990s, peak yellow-sticky trap catch exceeded 20 adults per trap per day (Blua et al. 1999). Subsequently, within 3 years of the first reports of newly diseased vines in Temecula, CA in 1997, numerous vineyards had PD prevalence in excess of 50\% (Hopkins and Purcell 2002; Perring et al. 2001). In recent years, $H$. vitripennis abundance and PD pressure have been reduced due to area-wide and local integrated pest management, which includes biological and chemical control of vectors and roguing of diseased vines (Daugherty et al. 2015). Nonetheless, H. vitripennis remains a significant risk to grape production in areas where it is established 
should its populations rebound, and in areas where it has not yet invaded. To mitigate future impacts, an improved understanding is needed of the mechanism by which it is able to drive PD epidemics.

$H$. vitripennis is not as efficient at transmitting $X$. fastidiosa to grapevines as are other vectors (Almeida and Purcell 2003; Daugherty and Almeida 2009). Instead, H. vitripennis's role in epidemics stems from an ability to achieve high population densities or other aspects of its ecology and behavior that fundamentally alter $X$. fastidios $a$ transmission and spread compared with other vectors (Almeida et al. 2005a). In coastal vineyards, $G$. atropunctata activity is related to seasonal migration from nearby reproductive habitat, with peaks in activity occurring in late spring, smaller peaks in midsummer, and little activity the rest of the year (Purcell 1975). This results in patterns of disease that are consistent with primary spread from neighboring breeding habitats (Purcell 1974). Conversely, H. vitripennis can be active in vineyards throughout the year (Blua et al. 2001), especially in late summer, and during the dormant season in vineyards adjacent to reproductive habitat (Blua and Morgan 2003). H. vitripennis is also more willing to feed on mature portions of vines (Almeida and Purcell 2003) and is able to transmit to dormant vines (Almeida et al. 2005b). This combination of a longer seasonal window of activity and ability to transmit year round led to the hypothesis that $H$. vitripennis may be capable of vine-to-vine (i.e., secondary) spread of $X$. fastidiosa (Almeida et al. 2005a; Hill et al. 2006). With vine-to-vine spread, prior (i.e., primary) infections contribute to secondary cases of disease in addition to any new primary infections. Implicitly, more severe disease epidemics should be possible (or occur at lower vector densities) for pathosystems with secondary versus strictly primary pathogen spread (Almeida et al. 2005a). Thus, quantifying the ability of $H$. vitripennis to promote vine-to-vine spread may yield important insights into its epidemiological significance.

Field surveys have documented clustering of PD cases, which may be indicative of secondary spread by $H$. vitripennis (Park et al. 2006), whereas others found a majority of blocks with patterns of disease that were more consistent with primary spread (Park et al. 2011). One explanation for why patterns of disease could vary relates to the role of seasonality in PD epidemiology. Multiple sources of seasonality exist in the PD system that may affect the ability of $H$. vitripennis to promote vine-to-vine spread (Hill et al. 2006). One source stems from variability in $X$. fastidiosa infection dynamics. In the field, infection levels and disease symptoms vary over the season, with low infection levels during the early season and greater infection levels and symptoms later in the season (Hopkins 1981; Hopkins and Thompson 1984). This is consistent with detectability assays, which show a greater fraction of known infected vines testing positive later in the season (Hill et al. 2006). Seasonal infection levels may be important given that sharpshooter acquisition depends on infection level in the plant, with thresholds below which acquisition rarely occurs (i.e., $<10^{4} \mathrm{CFU} / \mathrm{g}$ of plant) (Hill and Purcell 1997). In other words, low X. fastidiosa infection levels in vines early in the year may limit or delay the potential for those vines to be sources of infection for secondary cases. A second source of seasonality stems from the recovery or "cold curing" of X. fastidiosa-infected vines over the winter (Purcell 1977). Recovery rate is affected by winter temperatures, grapevine cultivar, and the time of year vines were inoculated (Feil et al. 2003; Hill et al. 2006; Lieth et al. 2011). With respect to inoculation timing, early-season infections (e.g., prior to summer) are far less likely to lead to host recovery over the winter than are late-season infections (Feil et al. 2003).

We studied $H$. vitripennis-mediated vine-to-vine spread to clarify this vector's ability to drive PD epidemics. First, we developed a general disease model that captures the salient seasonal features of the PD system to understand their implications for disease dynamics. We used the model to evaluate broadly the hypothesis that seasonality in acquisition and recovery constrains disease incidence, rather than to generate specific predictions about how much incidence is reduced. Next, we established a field study to determine whether $H$. vitripennis promotes vine-to-vine spread and search for patterns in incidence that support the conclusions from the modeling regarding the epidemiological importance of seasonality in the PD pathosystem.

\section{MATERIALS AND METHODS}

Modeling disease dynamics with seasonal effects. Despite more than 100 years of research on PD epidemiology (Pierce 1892), few formal models of its dynamics have been developed. The handful of models that exist include general analytic epidemiological compartment models focused on vector behavior that are implicitly relevant to the PD pathosystem (Zeilinger and Daugherty 2014), general spatially explicit simulation models that are implicitly relevant to PD management (Sisterson and Stenger 2013), and an analytic model more specific to PD that evaluated different control strategies (Kyrkou et al. 2018). Yet, to date, models have largely ignored the role that seasonality may play in PD dynamics.

To understand the epidemiological significance of seasonality in the PD system, we developed a general, discrete-time simulation model of coupled "susceptible-infected" vector-host infection dynamics (Appendix 1). A simulation approach was used because it more conveniently allows for the inclusion of seasonally variable, nonlinear values of key model parameters, particularly those relating to acquisition and overwinter recovery rates (Appendix 2). Throughout, we assumed a monthly timestep $(t)$ to capture broadly seasonal differences in these parameters, with recovery occurring at the end of each 12-month period.

In the model, the state variables $S, I, U$, and $V$ equate to the density of healthy host plants, infected plants, noninfective vectors, and infective vectors, respectively. Both constant host $(S+I=N)$ and vector $(U+V=T)$ population densities were assumed, which allows for a complete description of model dynamics via the following paired difference equations:

$$
\begin{gathered}
V_{i+1}=V_{t}+c a_{m}\left(T-V_{t}\right) I_{t} / N-d V_{t} \\
I_{i+1}=I_{t}+c b\left(N-I_{t}\right) V_{t} / N-R
\end{gathered}
$$

where $c$ is the vector feeding (i.e., biting or attack) rate, $a_{m}$ is acquisition rate (i.e., proportion of noninfective vector contacts with infected hosts that result in pathogen acquisition), $d$ is vector turnover (i.e., mortality or migration) rate, $b$ is inoculation rate (i.e., proportion of infective vector contacts with healthy hosts that result in pathogen inoculation), and $R$ is the between-year recovery of infected hosts back to the susceptible state, which is defined by the following term:

$$
R=\sum_{m=\mathrm{Jan}}^{\mathrm{Dec}} r_{m} c b\left(N-I_{t}\right) V_{t} / N
$$

where $r_{m}$ denotes host recovery rate (the proportion of infected hosts inoculated in a given month that lose infection at the end of the year). Hereafter, we refer to newly infected host plants as "acute infections" or "acute spread", versus "chronic infections" or "chronic spread". The two terms acute and chronic are needed to capture the fact that, in the PD pathosystem, newly infected vines may or may not recover over the winter. Recovery of host plants infected early in the season is low; therefore, early infections are expected to persist after the first winter and to lead to chronic disease. Conversely, late-season infections are associated with high recovery rate and acute disease.

Throughout, vector feeding rate and inoculation rate were assumed to be constant over time, which may not be the case if vectors respond to different host phenotypes (Sisterson 2008) or if vector activity levels or feeding rates in a focal habitat vary seasonally (Purcell 1974). Vector turnover rate reflects loss due to 
mortality or emigration and was also assumed to be constant which, coupled with a constant vector population $(T)$, is equivalent to assuming that all vectors that emigrate or die are replaced by noninfectious ones via reproduction or immigration.

In our simulations, sharpshooter acquisition rate of $X$. fastidiosa was assumed to vary monthly based on observations from the PD system that (i) infection levels in plants vary seasonally (Hill et al. 2006; Hopkins 1981) and (ii) sharpshooter acquisition efficiency is related to infection level (Hill and Purcell 1997). Although we considered differences in the magnitude of acquisition, we typically assumed a similar nonlinear form for the monthly acquisition rate as described by observations of $X$. fastidiosa detectability by Hill et al. (2006); low until late spring, then increasing through much of summer. We also assumed that acquisition declined substantially with the onset of leaf-fall (Supplementary Fig. S1; Fig. 1B). Finally, our analyses emphasized comparison of disease dynamics under scenarios of seasonally varying acquisition to constant acquisition over time, with the constant rate calculated as the average of the monthly seasonal rates (e.g., seasonal: $a_{m}=0.01$ to 0.5 , versus constant: $a_{m}=0.22$ ) (Fig. 1).

We assumed host overwinter recovery that approximates observations from field studies (Feil et al. 2003; Hill et al. 2006), which showed low recovery rates for plants infected in the spring, increasing values over spring into summer, and high values for infections occurring summer through fall. Because the precise timing of when vines recover over the winter is not well defined, we assumed it occurred at the end of each 12th month (i.e., between December and January) as a matter of convenience (Supplementary Fig. S2; Fig. 1B). Simulations explored differences in the magnitude of recovery, as might occur due to differences in winter
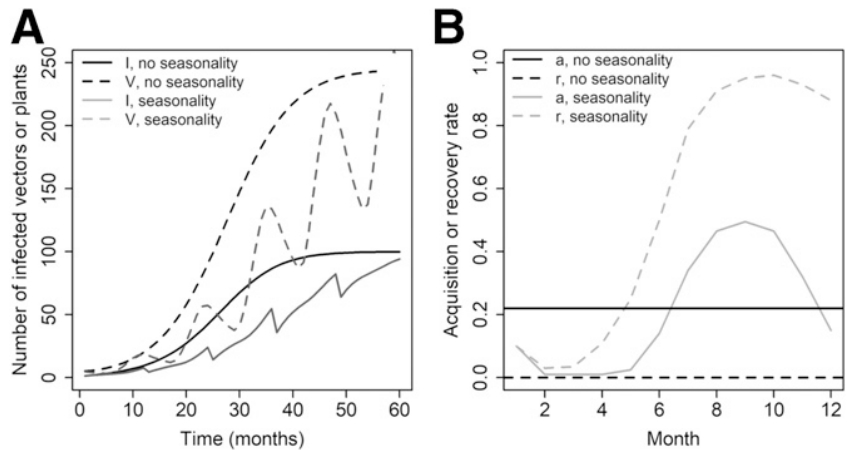

Fig. 1. A, Prevalence of infected host plants $(I)$ and vectors $(V)$ over time with and without seasonality in acquisition rate and host recovery. B, Parameter values used in simulations assuming seasonality versus no seasonality in acquisition and recovery rate, by month (January to December $=1$ to 12 ). Vector population $=500$, host population $=100$, initial infected plants $\left(I_{0}\right)=1$, initial infective vectors $\left(V_{O}\right)=5$, vector feeding rate $=0.4$, inoculation rate $=0.25$, vector turnover $=0.1$, and acquisition rate $\left(a_{m}\right)$ and host recovery rate $\left(r_{m}\right)$ were either constant or varying monthly. severity or among cultivars (Lieth et al. 2011), with an emphasis on comparing scenarios with seasonal recovery to those without recovery (e.g., seasonal: $r_{m}=0.03$ to 0.95 , versus constant: $r_{m}=0$ ) (Fig. 1 ).

Because our objective was to describe generally the influence of seasonality on model behavior (versus predicting specific outcomes), along with uncertainty regarding the specific value of certain parameters or parameters that might be inherently variable in the field, we explored disease dynamics over a range of parameter space (Table 1). As noted, simulations focused especially on comparing disease dynamics without seasonal acquisition or recovery to simulations with no seasonal effects. We also used sensitivity analysis to characterize broadly the effect of specific parameters on disease dynamics and to explore interactive effects among parameters. First, we analyzed disease dynamics in hosts and vectors given intermediate assumptions regarding seasonal acquisition ( $a_{m}=0.01$ to 0.5$)$ and recovery ( $r_{m}=0.03$ to 0.95$)$ versus constant acquisition $\left(a_{m}=0.22\right)$ and no recovery $\left(r_{m}=0\right)$. Second, we compared the dynamics of infected hosts given assumptions of no seasonality $\left(a_{m}=0.22, r_{m}=0\right)$, moderate seasonal acquisition $\left(a_{m}=0.01\right.$ to $\left.0.5, r_{m}=0\right)$, moderate seasonal recovery $\left(a_{m}=0.22, r_{m}=\right.$ 0.03 to 0.95$)$, or both sources of seasonality $\left(a_{m}=0.01\right.$ to $0.5, r_{m}=$ 0.03 to 0.95 ). Next, we analyzed how seasonality influenced the timing of disease incidence over the year by comparing the number of newly infected hosts produced during each month over the first year of an epidemic under scenarios with no seasonality $\left(a_{m}=0.22\right.$, $\left.r_{m}=0\right)$ versus seasonal acquisition and recovery $\left(a_{m}=0.01\right.$ to 0.5 , $r_{m}=0.03$ to 0.95 ). We also conducted sensitivity analysis on the magnitude of seasonal parameters, including overall acquisition rate ( $a_{m}=0$ to $0.25, a_{m}=0.01$ to 0.5 , or $a_{m}=0.02$ to $0.75 ; r_{m}=0.03$ to $0.95)$ and overall host recovery $\left(r_{m}=0\right.$ to $0.85, r_{m}=0.03$ to 0.95 , or $r_{m}=0.1$ to $1 ; a_{m}=0.01$ to 0.5$)$. Next, we conducted sensitivity analyses over a range of constant values for model parameters in the absence of seasonality $\left(a_{m}=0.22, r_{m}=0\right)$ versus with seasonal acquisition and recovery $\left(a_{m}=0.01\right.$ to $0.5, r_{m}=0.03$ to 0.95$)$. This included exploring the effects of parameters associated with vector transmission efficiency, such as feeding rate ( $c$, range: 0.3 to 1$)$ and inoculation rate ( $b$, range: 0.2 to 0.6$)$, and parameters that are relevant to management such as vector turnover rate ( $d$, range: 0.1 to 0.3 ) and vector total population density ( $T$, range: 100 to 1,000$)$. Finally, we simulated disease dynamics using values of key parameters estimated in our field experiment described below, in which the frequency of acute pathogen spread over the season $(0.21$ to 0.57$)$ was used as a proxy in estimating seasonal acquisition rate $\left(a_{m}, 0.04\right.$ to 0.62$)$ and the observed proportion of acute secondary infections that were lost over the winter ( 0 to 0.42$)$ as an estimate of recovery $\left(r_{m}, 0\right.$ to 0.75$)$. We compared disease dynamics with and without $\left(a_{m}=0.29, r_{m}=0\right)$ seasonal acquisition and host recovery at different vector densities (total vector density, $T=750$ or 250 ) and at different vector efficiencies (inoculation rate, $b=0.5$ or 0.25 ). For all simulations, we explored a range of initial conditions and durations of time but focused on scenarios where infected hosts and

TABLE 1. Model parameters, units, range of values used in simulations, and relevant references ${ }^{\mathrm{a}}$

\begin{tabular}{|c|c|c|c|}
\hline Parameter or variable & Units & Values & Reference \\
\hline Acquisition rate $\left(a_{m}\right)$ & Proportion & $\begin{array}{l}\text { Seasonal: } 0-0.75 \\
\text { Constant: } 0.22,0.29\end{array}$ & Hill et al. 2006 \\
\hline Inoculation rate $(b)$ & Proportion & $0.2-0.6$ & Daugherty and Almeida 2009 \\
\hline Feeding rate $(c)$ & Dimensionless & $0.3-1$ & - \\
\hline Vector turnover rate $(d)$ & Proportion & $0.1-0.3$ & - \\
\hline Recovery rate $\left(r_{m}\right)$ & Proportion & $\begin{array}{l}\text { Seasonal: } 0-1 \\
\text { Constant: } 0\end{array}$ & Feil et al. 2003, Hill et al. 2006 \\
\hline Host plant density $(N)$ & Number/area & $100-500$ & - \\
\hline Vector density $(T)$ & Number/area & $200-1,000$ & - \\
\hline Initial infected plants $\left(I_{O}\right)$ & Number/area & $\leq 5 \%$ of $N$ & - \\
\hline Initial infected vectors $\left(V_{O}\right)$ & Number/area & $\leq 5 \%$ of $T$ & - \\
\hline Time duration (January start) & Months & $12-60$ & - \\
\hline
\end{tabular}

${ }^{a}$ Ranges of values were used because published estimates are not available or the parameter is expected to be inherently variable. 
vectors were initially rare (e.g., $I_{O}$ and $V_{O} \leq 5 \%$ ), and over agriculturally relevant timescales (e.g., $\leq 5$ years). All simulations were run in the $\mathrm{R}$ programming language (v.3.4.1; R Foundation for Statistical Computing, Vienna).

Estimating the magnitude of vine-to-vine spread. We established a replicated, 2-year field cage experiment to quantify $H$. vitripennis-mediated vine-to-vine spread in light of potential constraints stemming from seasonal effects in the PD pathosystem. Because it was not possible to compare experimentally disease dynamics in seasonal versus nonseasonal contexts (unlike in the model), our goal was not to validate specific outcomes in the modeling. Rather, we used the experiment to determine whether $H$. vitripennis promoted vine-to-vine spread and to define patterns in disease incidence that are relevant to understanding whether seasonality affects vine-to-vine spread. This included testing the following predictions: (i) risk of acute pathogen spread increases over the season, (ii) recovery is more likely for those vines infected later in the season, and (iii) risk of chronic PD spread varies over the season.

In early spring 2007, 48 insect-proof cages ( 1.75 by 1.75 by $2 \mathrm{~m}$, 21 by 21 threads/cm; Synthetic Industries Inc., Alto, GA) were set up in a research plot adjacent to the University of California Cooperative Extension offices in Bakersfield. Cage walls were sunk under the soil surface by at least $10 \mathrm{~cm}$. Within each cage we planted five grapevines of one of two table grape cultivars ( $V$. vinifera 'Thompson Seedless 2A' or 'Redglobe 02'; Sunridge Nurseries, Bakersfield, CA). These two cultivars were selected because they are commonly grown in the region and differ in susceptibility to infection by $X$. fastidiosa (Krell et al. 2008). Vines were dormant bench grafts at the time of planting, and were evenly spaced within cages such that one vine was near each corner and one was planted at the center.

The following spring, in April 2008, we randomly selected one vine per cage for mechanical inoculation of two canes, using a pinprick method after pipetting $10-\mu \mathrm{l}$ droplets of a turbid suspension of $X$. fastidiosa subsp. fastidiosa in succinate-citrate-phosphate buffer ("Stags Leap" [STL] isolate) (Hill and Purcell 1995). Flagging was used to mark the approximate inoculation points. We then introduced into the cages cohorts of $15 \mathrm{H}$. vitripennis adults from a pathogen-free colony established earlier in the season, primarily from field-collected egg masses and nymphs from the Bakersfield, $\mathrm{CA}$. The sharpshooters were introduced at one of three times over the summer (early June, mid-July, or early September) and were allowed to feed and move for 10 days, after which we applied a foliar insecticide to kill the insects (Sevin; Techpac LLC, Atlanta Georgia). These three discrete windows of vector activity in cages were used to estimate the relative potential for $X$. fastidios $a$ spread at different times over the season. There were eight replicate cages for each vector introduction date and grapevine cultivar combination. In early November 2008, we collected up to five petiole or stem samples from all needle-inoculated and noninoculated (hereafter "initially healthy") vines in the experiment, which were returned to the lab for storage at $-20^{\circ} \mathrm{C}$. For needle-inoculated vines, we collected petiole and stem sections from those canes that had been inoculated at locations distal to the inoculation points. For initially healthy vines, samples were collected randomly from throughout at least three canes. If PD symptoms were apparent, tissue from those regions of the vine were prioritized for collection. Over the winter, all vines were pruned conservatively, leaving at least approximately $0.5 \mathrm{~m}$ of all canes (i.e., distal to inoculation points on needleinoculated vines). Finally, in late September 2009, we collected petioles from all vines in the same manner as the year prior.

Samples from all vines in fall 2008 were assayed for $X$. fastidiosa infection to confirm infection in needle-inoculated vines and to estimate the frequency of acute secondary spread to initially healthy vines. Infection in needle-inoculated vines, for which the inoculation point was known, was confirmed via polymerase chain reaction (PCR) of a single pooled sample using protocols described by Costa et al. (2004). Conversely, for 2008 samples from initially healthy vines, infection was confirmed with redundant assays. Because the point of inoculation by insects (if there was one) was not well defined, we first processed several samples from throughout each of the 192 initially healthy vines (nearly 5 on average; 948 samples total) via enzyme-linked immunosorbent assay (ELISA) (Hill et al. 2006) to increase the chance of $X$. fastidiosa detection if an infection had occurred. Although lab studies suggest that PCR can detect 100-fold lower numbers of $X$. fastidiosa cells than ELISA (Minsavage et al. 1994), detection rates on field-collected samples suggest that the two methods perform equivalently (Amanifar et al. 2014; Bextine and Miller 2004). Nonetheless, given the potential for cross reactivity in ELISA to result in false positives (Costa et al. 2004), we reassayed all ELISA-positive initially healthy vines in 2008 via a single pooled sample using PCR. In all cases, samples consisted of approximately equal fractions of pooled subsamples from at least two petioles from different canes on the same vine. For a minority of vines (approximately 25\%), there was insufficient intact petiole material present at the time of collection, due to marked leaf senescence associated with the onset of dormancy at the time of collection; therefore, we assayed samples from collected shavings made with a razor blade from the exterior portion of two to three stem sections. Samples from all vines in fall 2009 were assayed to estimate the number of acute secondary infections in 2008 that recovered over winter, versus those that developed into chronic infections. For all 2009 samples, X. fastidiosa infection status was assayed via PCR of pooled petiole samples.

Although we established 48 replicate cages, 8 were excluded from analysis because of poor growth of vines, the needleinoculated vine was never confirmed to be infected or was dead by the end of the first year, or insecticide treatments did not kill all $H$. vitripennis. This left six to eight replicate cages for each combination of vector introduction date and grape cultivar. We analyzed the frequency of acute spread of $X$. fastidios $a$ from needleinoculated to initially healthy vines at the end of the first year in two ways. First, we compared the number (out of four total per cages) of initially healthy vines testing positive in the first year using a generalized linear model (GLM) (Crawley 2009), with a fixed effect of grape cultivar, vector introduction date as a continuous variable, and zero-inflated Poisson error to appropriately capture the count nature of the response variable with frequent zeros ('zeroinfl()' in $\mathrm{R}$ package 'pscl') (Jackman 2017). Next, we analyzed whether any of the initially healthy vines in a given cage were infected at the end of the first year using a GLM, with a fixed effect of cultivar, introduction date as a covariate, and binomial error (Crawley 2009). We then analyzed how often acute infections at the end of the first year no longer tested positive in the second year as an estimate of overwinter recovery rate. These data were analyzed with a generalized linear mixed-effects model (GLMM), with cultivar and introduction date as fixed effects, cage identity as a random effect to account for autocorrelation among vines within the same cage, and binomial error (Pinheiro and Bates 2000). A significant effect of introduction date was followed up by pairwise binomial GLMMs between introduction dates, with Bonferroni correction to account for multiple comparisons (Crawley 2009). Finally, we analyzed the number of vines (out of five total per cage) that were infected at the end of the second year as a reflection of the frequency chronic infection, using a Poisson GLM with a fixed effect of cultivar and introduction date as a covariate. For all analyses, we employed $\chi^{2}$ tests to compare the fit of a maximum model to a series of simpler models, with interaction terms or main effects removed, to determine the most parsimonious, adequate model (Crawley 2009). All analyses were conducted in the $\mathrm{R}$ programming language, v.3.4.1.

\section{RESULTS}

Modeling disease dynamics with seasonal effects. Overwinter recovery at the end of each season induced cyclic dynamics 
into the prevalence of infected hosts compared with more monotonic increases in prevalence in the absence of seasonality (Fig. 1). Then, as long as some vector mortality or emigration occurred (i.e., $d>0$ ), the prevalence of infected vectors showed similar cyclic behavior due to a reduced number of acquisition opportunities early in the season and increased number late in the season (Fig. 1A). Compared with scenarios with constant acquisition and no recovery, seasonal acquisition alone slightly delayed disease prevalence, and seasonal recovery alone generally resulted in more substantial overall reduction in disease prevalence (Fig. 2). Reductions in disease prevalence are most pronounced when both sources of seasonality occurred, suggesting interactive effects of seasonal acquisition and recovery (Fig. 2).

The apparent potential for seasonality to constrain disease incidence occurred over a wide range of parameter space (Appendix 3 ), with its extent depending on assumptions regarding the magnitude of seasonal acquisition (Supplementary Fig. S3) and overwinter recovery (Supplementary Fig. S4). Higher seasonal acquisition rates and lower recovery rates were associated with greater incidence. In general, when both sources of seasonality occurred, disease dynamics appeared more sensitive to the relative magnitude of acquisition than recovery. The effect of seasonality also depended on aspects of the pathosystem related to vector efficiency (Supplementary Fig. S5) and vector pressure (Supplementary Fig. S6). Although seasonality increased the range of parameter space over which relatively low disease incidence occurred, there remained significant potential for efficient vectors (i.e., high inoculation, $b$, and feeding rates, $c$ ) or high vector pressure (i.e., high vector population density, $T$, or low vector turnover, $d$ ) to promote severe disease epidemics.

The interaction between seasonal acquisition and recovery on disease incidence stemmed from their combined effect on the timing of seasonal windows of disease incidence (Fig. 3). In the absence of seasonality, the frequency of newly infected vines

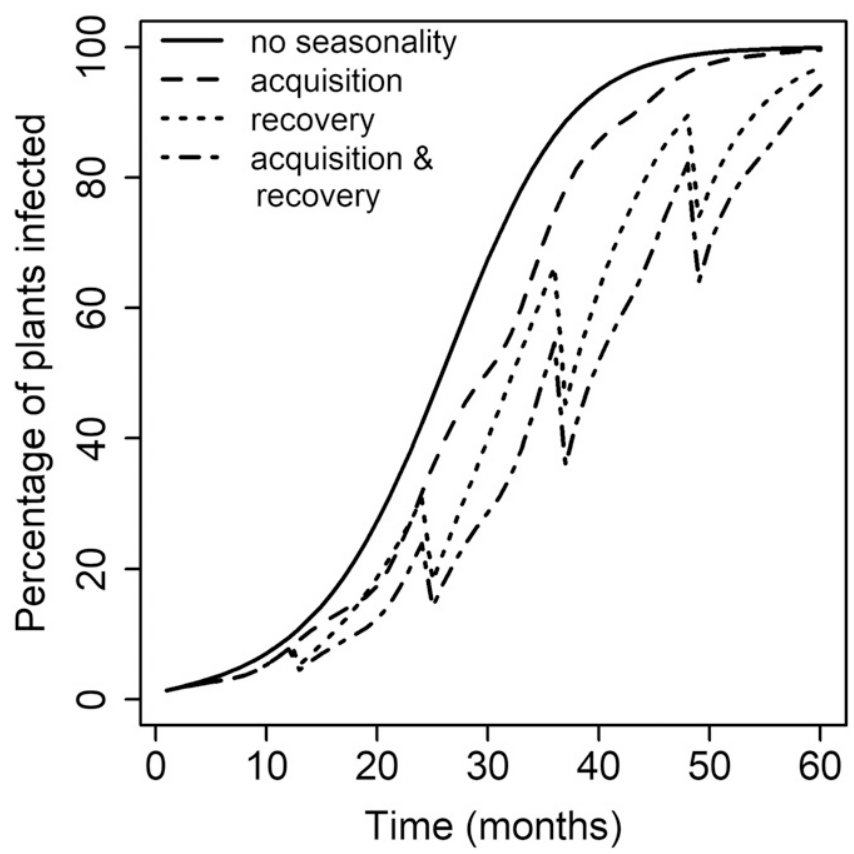

Fig. 2. Prevalence of infected plants over time with constant acquisition and no host overwinter recovery (i.e., no seasonality), seasonally variable acquisition but no host recovery, seasonal recovery but constant acquisition, or seasonal acquisition and seasonal recovery. Constant acquisition $\left[a_{m}\right]=0.22$, constant recovery rate [i.e., no recovery; $\left.r_{m}\right]=0$, seasonal acquisition $\left[a_{1}\right.$ to $\left.a_{12}\right]=0.03$, $0.03,0.04,0.05,0.08,0.16,0.32,0.44,0.48,0.46,0.33,0.13$, seasonal recovery $\left[r_{1}\right.$ to $\left.r_{12}\right]=0.03,0.01,0.03,0.09,0.3,0.56,0.76,0.89,0.95,0.97$, $0.98,0.97$. Vector population $=500$, host population $=100$, initial infected plants $=1$, initial infective vectors $=5$, vector feeding rate $=0.4$, inoculation rate $=0.25$, and vector turnover $=0.1$. increased over the season as both more vectors became infective and more hosts became infectious. Seasonal acquisition, as assumed to occur in the model, reduced incidence early in the year but exaggerated incidence later in the year compared with constant acquisition. However, although more new infections occurred later in the season, with recovery, few of those late-season infections persisted over the winter. This reduced the overall number of chronic infections and resulted in a temporal shift toward peak incidence from late season to early season (Fig. 3).

Our final analysis of disease dynamics drew upon values of seasonal acquisition and recovery from the field experiment. The frequency of any acute spread to initially healthy vines was fit to a two-parameter logistic function $\left(y=1 /\left[1+e^{-(x-a) b}\right]\right.$, where $a=$ $231.3 \pm 2.706$ and $b=0.017 \pm 0.002$ ) which, after extrapolation to the remainder of the year based on plausible expectations, resulted in estimates of monthly acquisition $\left(a_{m}\right)$ that ranged from 0.04 in midwinter to 0.62 in late summer (Fig. 4C) versus a constant mean acquisition of 0.29 . Overwinter recovery estimates from the field experiment were fit to the same logistic function $(a=249.788 \pm$ 0.059 and $b=0.074 \pm 0.001$ ), producing a range in the monthly proportion recovering from 0 to 0.75 (Fig. 4C) versus no recovery $\left(r_{m}=0\right)$. Simulations using these parameter values were largely congruent with earlier results, in that seasonality moderated disease incidence but also depended on other parameter values (Fig. 4). Differences in disease incidence between simulations with and without seasonality were more pronounced at high total vector population density $(T=750)$ than at relatively lower vector density $(T=250)$ (Fig. 4A), and at high vector efficiency $(b=0.5)$ compared with relatively lower vector efficiency $(b=0.25)$ (Fig. 4B).

Estimating the magnitude of vine-to-vine spread. Overall, 26 of 160 initially healthy vines (approximately 16\%) were infected by $X$. fastidios $a$ by the end of the first year, with nearly $40 \%$ of cages (15 of 40) having at least one secondary infection. For the

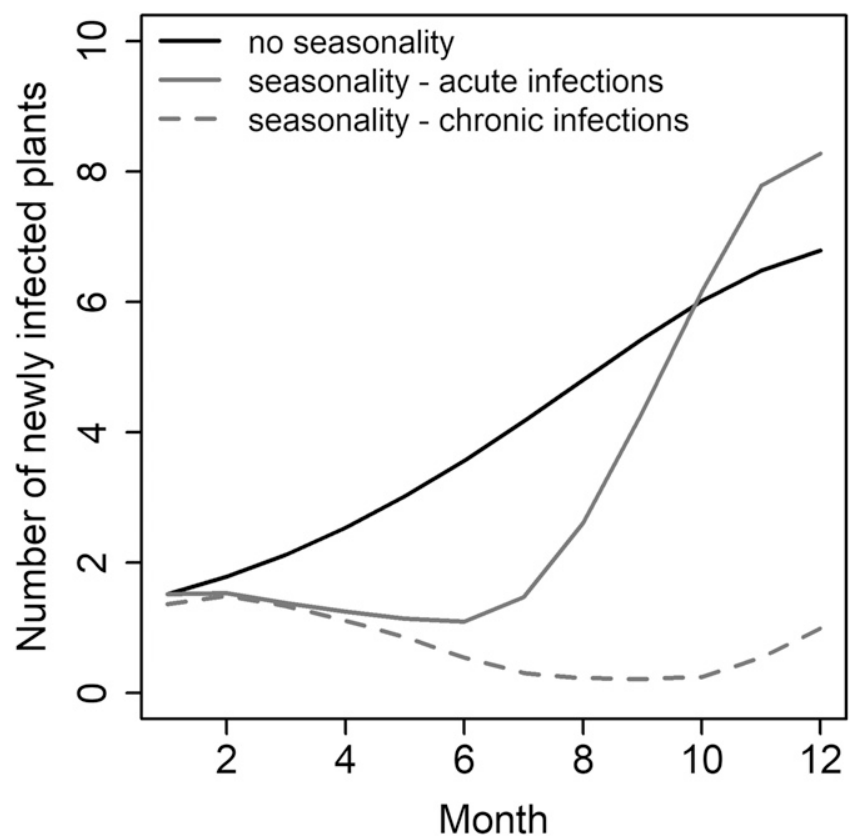

Fig. 3. Incidence of newly infected host plants in the first year of an epidemic, by month, with constant acquisition and no recovery (black line) versus seasonal incidence of newly infected hosts prior to recovery (acute cases; gray solid line) and after recovery (chronic cases; gray dashed line) if seasonal acquisition and recovery occur. Vector population $=1,000$, host population $=$ 100 , initial infected plants $=5$, initial infective vectors $=20$, vector feeding rate $=0.35$, inoculation rate $=0.3$, vector turnover $=0.2$, constant acquisition rate $\left[a_{m}\right]=0.22$, seasonal acquisition rate $\left[a_{1}\right.$ to $\left.a_{12}\right]=0.03,0.03,0.04,0.05$, $0.08,0.16,0.32,0.44,0.48,0.46,0.33,0.13$, constant recovery [i.e., no recovery; $\left.r_{m}\right]=0$, and seasonal host recovery rate $\left[r_{1}\right.$ to $\left.r_{12}\right]=0.03,0.01,0.03$, $0.09,0.3,0.56,0.76,0.89,0.95,0.97,0.98,0.97$. 
A
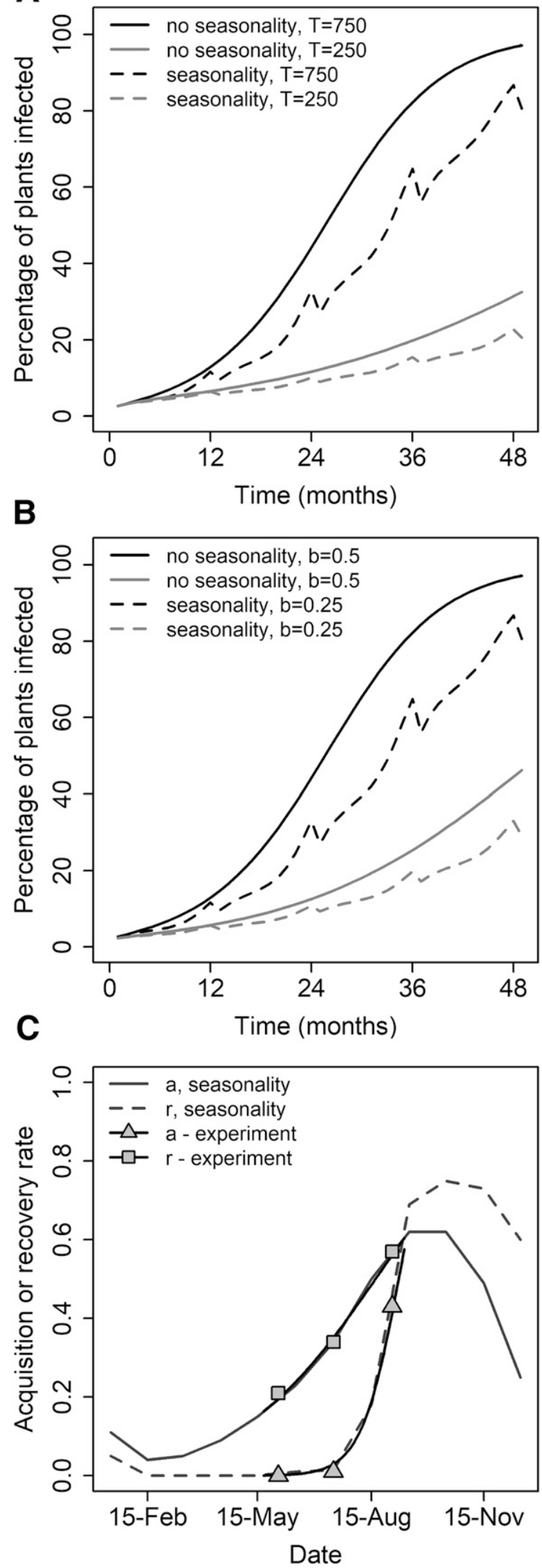

Fig. 4. Prevalence of infected hosts over time with and without (acquisition rate $\left[a_{m}\right]=0.29$ and host recovery rate $\left[r_{m}\right]=0$ ) seasonal acquisition and host recovery $\mathbf{A}$, at two levels of vector pressure (vector population $[T]=750$ or 250 and inoculation rate $[b]=0.5$ ) or $\mathbf{B}$, two levels of vector efficiency $(b=0.5$ or 0.25 , $T=750)$. C, Monthly values of seasonal acquisition $\left(a_{m}\right)$ and recovery $\left(r_{m}\right)$ using extrapolation (gray lines) of observed values from the field experiment (symbols and black lines). Host population $=250$, initial infected plants $=5$, initial infective vectors $=10$, vector feeding rate $=0.35$, and vector turnover $=0.25$. number of new infections per cage, the final model included only an effect of vector introduction date-the effect of cultivar and the interaction were dropped during model simplification. Although there were greater numbers of new infections at later vector introduction dates (Fig. 5A), this trend was not significant $\left(\chi^{2}=2.609, \mathrm{df}=1, P=0.1061\right)$. For the frequency of cages with at least one new infection, the final model included only a significant effect of vector introduction date $\left(\chi^{2}=3.915\right.$, $\mathrm{df}=1, P=0.0475$ ), with nearly a threefold increase in the frequency of infections between the first and third introduction dates (Fig. 5B).

Of the new infections that occurred in first year, overall, approximately $27 \%$ no longer tested positive at the end of the second season. The final model for frequency of recovery included only a significant effect of vector introduction date $\left(\chi^{2}=9.355\right.$, $\mathrm{df}=2, P=0.0093)$. None of the acute infections from the first two vector introductions recovered, versus over $40 \%$ of those from the final vector introduction (Fig. 6).

The number of vines (out of 5 per cage) infected at the end of the second year ranged between 0 and 4, with an overall mean of 1.175 per cage. The final model included only a nonsignificant effect of introduction date $\left(\chi^{2}=1.636\right.$, df $\left.=1, P=0.2009\right)$. The first two introduction dates had similar numbers of chronic infections: on average, slightly $>1$ (i.e., the number of primary infections). For the final introduction date, there was a lower number of chronic infections, though not significantly $<1$ (Fig. 7).

\section{DISCUSSION}

Successful management outcomes are often favored by the application of multiple management tactics (Jeger et al. 2004). Nonetheless, estimates of the relative importance of primary versus secondary spread of a pathogen can inform expectations about the relative value of specific tactics such as vector control (Perring et al. 1999) or removal of diseased hosts (i.e., roguing) (Cooper et al. 2018). Here, we studied the ability of an invasive vector to promote secondary spread to understand its role in PD epidemics. This included evaluating the potential for seasonality in the PD pathosystem to limit disease incidence.

Results from our modeling largely confirm the hypothesis that seasonality in the PD pathosystem may moderate vine-to-vine disease incidence (Hill et al. 2006). The moderating influence stems, in part, from low early-season acquisition, which limits or shifts the temporal window for spread of primary cases of disease, particularly if there is some turnover in vector populations. In effect, it delays the potential for acute secondary spread to occur until later in the season, when additional plants become infected and are also higher-quality sources of $X$. fastidiosa. This pattern is supported by preliminary data on natural infectivity of $H$. vitripennis in the field, which increased from $<10 \%$ positive for $X$. fastidiosa at the end of spring to $>60 \%$ at the end of fall but then reset to low levels the following season (M. P. Daugherty, unpublished data). Any delay in acute spread is important for disease incidence because it accentuates overwinter recovery (Feil et al. 2003). Recovery has been observed for $X$. fastidiosa infections in other hosts (Ledbetter et al. 2009) and phytoplasma diseases of grapevines (Bellmo et al. 2007; Morone et al. 2007). For PD, Feil et al. (2003) estimated that approximately half of infections occurring at the end of April recovered over the winter, which increased to $>90 \%$ of infections that occurred at the end of June. Hill et al. (2006), who studied different cultivars at a warmer site, found lower recovery but a similar seasonal trend. Our modeling included the simplifying assumption of constant vector densities over time. In reality, though $H$. vitripennis is active to some degree throughout the year, its abundance or activity are typically higher, in some cases orders of magnitude higher (Blua and Morgan 2003), in mid- to late summer than earlier in the season (Blua et al. 2001). Thus, the majority of inoculation events are likely to occur later in the season as compared with model assumptions. If so, our results may be a conservative reflection of the effect of seasonality on chronic vine-to-vine spread. 
The conclusion that seasonality moderates vine-to-vine spread of $\mathrm{PD}$ is congruent with observations of PD epidemiology on the north coast of California. An analysis of $G$. atropunctata abundance, natural infectivity, and recovery estimated that, together, these sources of seasonality may reduce PD incidence up to eightfold compared with if they were not present (Gruber and Daugherty 2013). Ultimately, the extent to which seasonality in acquisition and recovery limit chronic vine-to-vine PD spread depends on their magnitude and precise nature relative to each other. $X$. fastidiosa multiplication rate depends on temperature (Feil and Purcell 2001), recovery rates depend on the severity of winter temperatures (Lieth et al. 2011), and both infection levels (Rashed et al. 2013) and recovery may differ among grape cultivars (Lieth et al. 2011). Future studies of the interplay between climate and varietal differences seem worthwhile for clarifying the contexts in which vine-to-vine spread is most likely.

Vector-pathogen transmission mode and other epidemiological characteristics can influence disease dynamics, with implications for disease management (Madden et al. 2000). For example, in cases
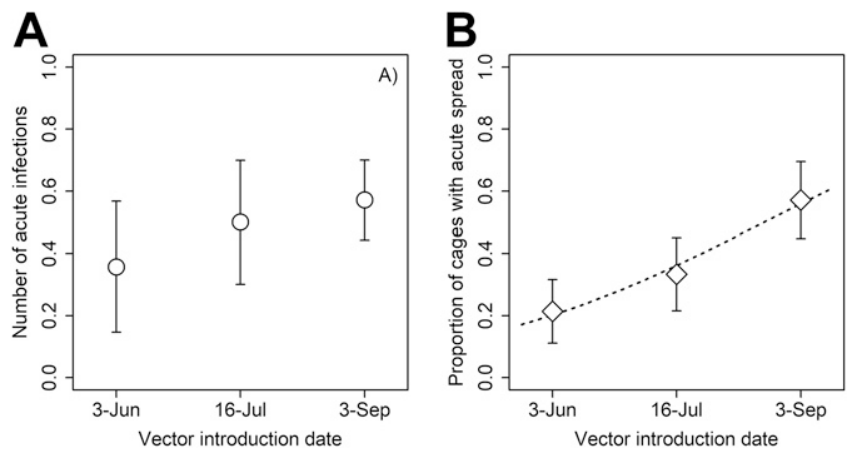

Fig. 5. A, Mean ( \pm standard error $[S E]$ ) number of initially healthy vines per cage infected by Xylella fastidiosa and $\mathbf{B}$, the proportion $( \pm$ SE) of cages with any new infections by the end of the first season based on the time of year vectors were introduced. The dotted line (B) depicts the model fit for a significant effect of vector introduction date.

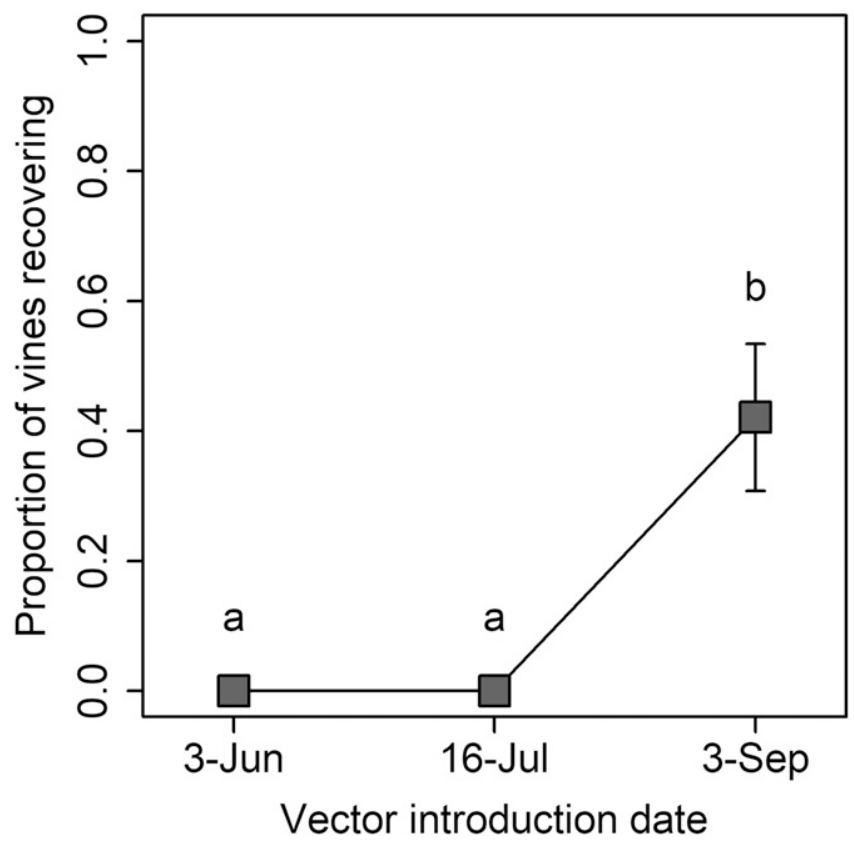

Fig. 6. Proportion ( \pm standard error) of infected plants in the first year that were not infected by Xylella fastidiosa at the end of the second year. Different letters above symbols denote significant differences between introduction dates. where pathogen spread is largely primary in nature, relying on insecticide applications for vectors in a focal plot rarely leads to successful outcomes (Perring et al. 1999). By moderating disease incidence in the PD pathosystem, seasonality should result in more modest requirements for reductions in vector pressure to curb pathogen spread-particularly for relatively inefficient vector species. Seasonality may also influence the utility of disease management tactics such as roguing, which seeks to minimize secondary spread by limiting acquisition opportunities for noninfective vectors. The effectiveness of roguing is likely to depend on characteristics of the pathosystem such as the relative rates of host incubation versus latency (Coletta-Filho et al. 2014), and on roguing practices in the surrounding landscape (Sisterson and Stenger 2013). Regardless, if roguing decisions are made prior to recovery, as is typically the case based on symptoms in the fall, some vines will be removed that would have recovered. This redundancy may reduce the need for and economic efficiency of roguing.

Results from our field experiment support generally the expected seasonal infection dynamics related to $H$. vitripennis mediated vine-to-vine spread. For example, the frequency of any acute spread occurring in a cage increased over the season (i.e., among vector introduction dates), which is consistent with field studies that show an increasing fraction of infected vines testing positive over the season (Hill et al. 2006). Similarly, we found that recovery rate was greater for the final vector introduction date compared with the first two dates, which is broadly consistent with patterns documented in prior studies of inoculated vines in the field that show higher rates of recovery for vines infected later in the season (Feil et al. 2003; Hill et al. 2006). Yet, we found little difference between grapevine cultivars in terms of both pathogen spread and recovery and, overall, modest recovery rates compared with other studies. The similar rates of pathogen spread in the first season could be explained by presumably similar acquisition on the two cultivars given that, despite differences in PD severity, they have similar infection levels (Krell et al. 2008). The modest recovery rates that we estimated may be attributable to a relatively limited number of acutely infected vines in our study, fewer inoculation dates, different grape cultivars, or a warmer climate at our site compared with one of those studies

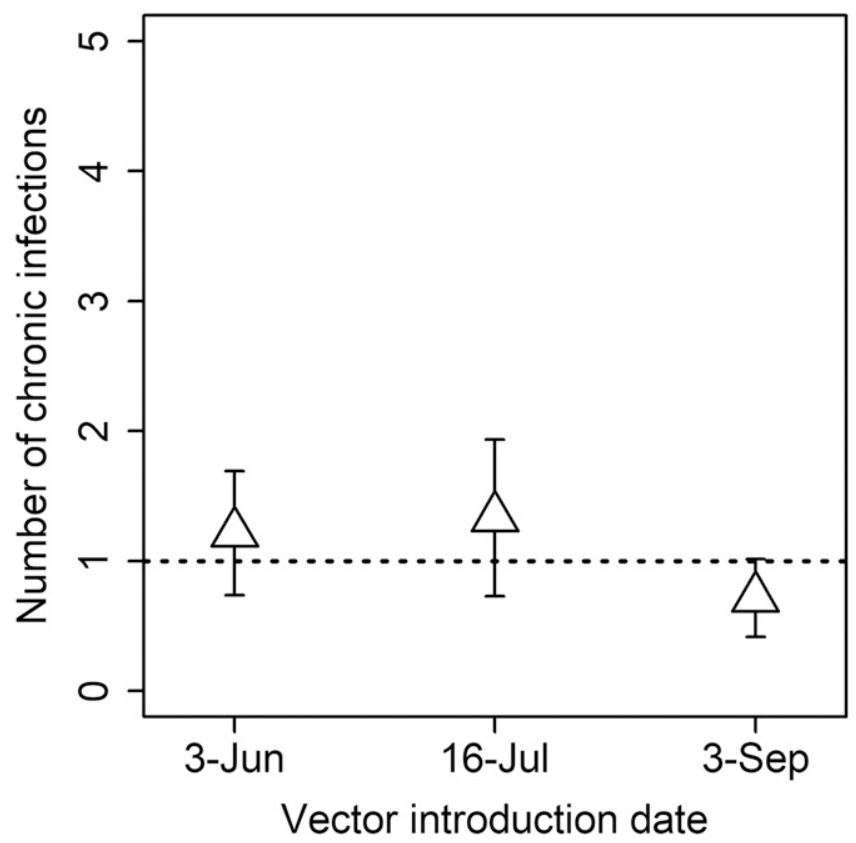

Fig. 7. Mean ( $\pm 95 \%$ confidence interval) number of vines infected by Xylella fastidiosa at the end of the second year. The dotted line denotes the number of primary infections ( 1 of 5) at the outset of the study after mechanical inoculation with $X$. fastidiosa. 
(Feil et al. 2003). Nonetheless, the observed seasonal patterns of acute spread and recovery in the experiment support key assumptions or patterns of incidence from the model, supporting the conclusion that seasonality plays a role in the ability of $H$. vitripennis to promote vine-to-vine spread.

The results from our field experiment indicate, at most, modest vine-to-vine spread. Specifically, the final number of infected vines was not significantly greater than the number of primary cases (i.e., one) created via mechanical inoculation with $X$. fastidiosa. Indeed, for the final vector introduction date, the mean number of chronic infections was slightly less than one, apparently due to a small number of the primary infections recovering. This observed low rate of vine-to-vine spread supports the results of the modeling and prior analyses on the moderating influence of seasonality on PD incidence (Gruber and Daugherty 2013). Yet, the low overall rate of spread, particularly with respect to acute secondary infections $(16 \%$ of all initially healthy vines), is also consistent with $H$. vitripennis's relatively low transmission efficiency to grapevines compared with other vectors (Almeida and Purcell 2003). A reanalysis of prior transmission experiments estimated that $G$. atropunctata was more than 10 times more efficient than $H$. vitripennis for a given access period or number of vectors (Daugherty and Almeida 2009). Collectively, these results indicate that, although $H$. vitripennis may be capable of vine-to-vine spread, it may not be efficient in doing so in many contexts, suggesting that its ability to drive PD epidemics stems from other aspects of its ecology.

Studies have shown that $H$. vitripennis is not as efficient a vector of $X$. fastidiosa to grapevines as are other vectors (Almeida and Purcell 2003; Daugherty and Almeida 2009). H. vitripennis also does not respond differently to host disease symptoms relative to other sharpshooters (Daugherty et al. 2011), which could otherwise explain differences in disease incidence (Sisterson 2008). Furthermore, the present study suggests that other aspects of its epidemiological efficiency such as an ability to promote secondary spread may be nominally important. Arguably, the remaining explanation for $H$. vitripennis driving PD epidemics is its high population densities or activity levels in some contexts. This conclusion is supported by a study showing insignificant PD incidence (Daugherty et al. 2015) in locations where H. vitripennis trap catch was orders of magnitude lower than its peak approximately a decade after its initial invasion (Blua and Morgan 2003; Blua et al. 1999, 2001). It is also supported, anecdotally, by a lack of reports of disease incidence attributable to $H$. vitripennis in areas of California where there is not substantial acreage of its key reproductive hosts (i.e., citrus). Collectively, this suggests that H. vitripennis-mediated disease spread may be a manageable problem, with vector control playing an important role (Pilkington et al. 2005; Sisterson et al. 2008). Ongoing efforts to mitigate the impact of this invasive vector should include maintaining the effectiveness of existing control measures and development of new tools.

\section{ACKNOWLEDGMENTS}

We thank J. Hashim-Buckey and M. Prior for their help establishing and maintaining the field experiment; L. Bittleston, J. Chau, L. Fernandez, N. Killiny, and C.-W. Tsai for help in setting up the cages; D. Morgan for help with insect collection; B. Hill, S. Purcell, and B. Marnuttle for helpful discussion that motivated this research; and three anonymous reviewers for their constructive feedback on an earlier version of this manuscript.

\section{LITERATURE CITED}

Almeida, R. P. P., Blua, M. J., Lopes, J. R. S., and Purcell, A. H. 2005a. Vector transmission of Xylella fastidiosa: Applying fundamental knowledge to generate disease management strategies. Ann. Entomol. Soc. Am. 98: 775-786.
Almeida, R. P. P., and Purcell, A. H. 2003. Transmission of Xylella fastidiosa to grapevines by Homalodisca coagulata (Hemiptera: Cicadellidae). J. Econ. Entomol. 96:264-271.

Almeida, R. P. P., Wistrom, C., Hill, B. L., Hashim, J., and Purcell, A. H. 2005b. Vector transmission of Xylella fastidiosa to dormant grape. Plant Dis. 89:419-424.

Amanifar, N., Mohsen, T., Karamat, I., and Babaei, G. 2014. Isolation and pathogenicity of Xylella fastidiosa from grapevine and almond in Iran. Phytopathol. Mediterr. 53:318-327.

Anderson, P. K., Cunningham, A. A., Patel, N. G., Morales, F. J., Epstein, P. R., and Daszak, P. 2004. Emerging infectious diseases of plants: Pathogen pollution, climate change and agrotechnology drivers. Trends Ecol. Evol. 19:535-544.

Bellmo, C., Carraro, L., Ermacora, P., Pavan, F., Osler, R., Frausin, C., and Governatori, G. 2007. Recovery phenomena in grapevines affected by grapevine yellows in Friuli Venezia Giulia. Bull. Insectol. 60:235-236.

Bextine, B. R., and Miller, T. A. 2004. Comparison of whole-tissue and xylem fluid collection techniques to detect Xylella fastidiosa in grapevine and oleander. Plant Dis. 88:600-604.

Blua, M. J., and Morgan, D. J. W. 2003. Dispersion of Homalodisca coagulata (Hemiptera: Cicadellidae), a vector of Xylella fastidiosa, into vineyards in Southern California. J. Econ. Entomol. 96:1369-1374.

Blua, M. J., Phillips, P. A., and Redak, R. A. 1999. A new sharpshooter threatens both crops and ornamentals. Calif. Agric. 53:22-25.

Blua, M. J., Redak, R. A., Morgan, D. J. W., and Costa, H. S. 2001. Seasonal flight activity of two Homalodisca species (Homoptera: Cicadellidae) that spread Xylella fastidiosa in Southern California. J. Econ. Entomol. 94: 1506-1510.

Coletta-Filho, H., Daugherty, M. P., Ferreira, C., and Lopes, J. 2014. Temporal progression of 'Candidatus Liberibacter asiaticus' infection in citrus and acquisition efficiency by Diaphorina citri. Phytopathology 104:416-421.

Cooper, M. L., Daugherty, M. P., Jeske, D. R., Almeida, R. P. P., and Daane, K. M. 2018. Incidence of grapevine leafroll disease: Effects of grape mealybug (Pseudococcus maritimus) abundance and pathogen supply. J. Econ. Entomol. 111:1542-1550.

Costa, H. S., Raetz, E., Pinckard, T. R., Gispert, C., Hernandez-Martinez, R., Dumenyo, C. K., and Cooksey, D. A. 2004. Plant hosts of Xylella fastidiosa in and near southern California vineyards. Plant Dis. 88:1255-1261.

Crawley, M. J. 2009. The R Book. John Wiley and Sons, Chichester, UK.

Daugherty, M. P., and Almeida, R. P. P. 2009. Estimating Xylella fastidiosa transmission parameters: Decoupling sharpshooter number and feeding period. Entomol. Exp. Appl. 132:84-92.

Daugherty, M. P., O'Neill, S., Byrne, F., and Zeilinger, A. 2015. Is vector control sufficient to limit pathogen spread? Environ. Entomol. 44:789-797.

Daugherty, M. P., Rashed, A., Almeida, R. P., and Perring, T. 2011. Vector preference for hosts differing in infection status: Sharpshooter movement and Xylella fastidiosa transmission. Ecol. Entomol. 36:654-662.

Davis, M. J., Purcell, A. H., and Thomson, S. V. 1978. Pierce's disease of grapevines: Isolation of the causal bacterium. Science 199:75-77.

Feil, H., Feil, W. S., and Purcell, A. H. 2003. Effects of date of inoculation on the within-plant movement of Xylella fastidiosa and persistence of Pierce's disease within field grapevines. Phytopathology 93:244-251.

Feil, H., and Purcell, A. H. 2001. Temperature-dependent growth and survival of Xylella fastidiosa in vitro and in potted grapevines. Plant Dis. 85: 1230-1234.

Freitag, J. H. 1951. Host range of Pierce's disease virus of grapes as determined by insect transmission. Phytopathology 41:920-934.

Germar, E. F. 1821. Bermerkungen über einige Gattungen der Cicadarien. Mag. Entomol. 4:1-106.

Gruber, B. R., and Daugherty, M. P. 2013. Understanding the effects of multiple sources of seasonality on the risk of pathogen spread to vineyards: Vector pressure, natural infectivity, and host recovery. Plant Pathol. 62:194-204.

Hewitt, W. B., Frazier, N. W., Jacob, H. E., and Freitag, J. H. 1942. Pierce's disease of grapevines. Calif. Agric. Exp. Stn. Circ. 353:1-32.

Hewitt, W. B., and Houston, B. R. 1941. Association of Pierce's disease and alfalfa dwarf in California. Plant Dis. Rep. 25:475-476.

Hill, B. L., Hashim, J., and Peacock, W. 2006. The effect of dormant season survival of Xylella fastidiosa in grapevines on Pierce's disease epidemics in California. Pages 276-279 in: 2006 Pierce's Dis. Res. Symp. Proc.

Hill, B. L., and Purcell, A. H. 1995. Multiplication and movement of Xylella fastidiosa within grapevine and four other plants. Phytopathology 85: 1368-1372.

Hill, B. L., and Purcell, A. H. 1997. Populations of Xylella fastidiosa in plants required for transmission by an efficient vector. Phytopathology 87:1197-1201.

Hopkins, D. L. 1981. Seasonal concentration of the Pierce's Disease bacterium in grapevine stems, petioles, and leaf veins. Phytopathology 71:415-418.

Hopkins, D. L., and Purcell, A. H. 2002. Xylella fastidiosa: Cause of Pierce's disease of grapevine and other emergent diseases. Plant Dis. 86:1056-1066. 
Hopkins, D. L., and Thompson, C. M. 1984. Seasonal concentration of the Pierce's Disease bacterium in 'Carlos' and 'Welder' Muscadine grapes compared with 'Schuyler' bunch grape. HortScience 19:419-420.

Jackman, S. P. 2017. Package 'pscl', version 1.5.2. R programming language. Online publication. https://cran.r-project.org/web/packages/pscl/pscl.pdf

Jeger, M. J., Holt, J., van den Bosch, F., and Madden, L. V. 2004. Epidemiology of insect-transmitted plant viruses: Modeling disease dynamics and control interventions. Physiol. Entomol. 29:291-304.

Krell, R. K., Perring, T. M., Hashim-Buckey, J. M., and Pinckard, T. R. 2008. Susceptibility of Vitis vinifera L. cv. Redglobe and Thompson Seedless to Pierce's disease. Am. J. Enol. Vitic. 59:61-66.

Kyrkou, I., Pusa, T., Ellegaard-Jenson, L., Sagot, M.-F., and Hansen, L. H. 2018. Pierce's disease of grapevines: A review of control strategies and an outline of an epidemiological model. Front. Microbiol. 9:2141.

Ledbetter, C. A., Chen, J., Livingston, S., and Groves, R. L. 2009. Winter curing of Prunus dulcis cv 'Butte' $P$. webbii and their interspecific hybrid in response to Xylella fastidiosa. Euphytica 169:113-122.

Lieth, J. H., Meyer, M. M., Yeo, K.-H., and Kirkpatrick, B. C. 2011. Modeling cold curing of Pierce's disease in Vitis vinifera 'Pinot noir' and 'Cabernet Sauvignon', grapevines in California. Phytopathology 101:1492-1500.

Madden, L. V., Jeger, M. J., and van den Bosch, F. 2000. A theoretical assessment of the effects of vector-virus transmission mechanism on plant virus disease epidemics. Phytopathology 90:576-594.

Minsavage, G. V., Thompson, C. M., Hopkins, D. L., Leite, R. M. V. B. C., and Stall, R. E. 1994. Development of a polymerase chain reaction protocol for detection of Xylella fastidiosa in plant tissue. Phytopathology 84:456-461.

Morone, C., Boveri, M., Giosuè, S., Gotta, P., Rossi, V., Scapin, I., and Marzachì, C. 2007. Epidemiology of flavescence dorée in vineyards in northwestern Italy. Phytopathology 97:1422-1427.

Park, Y.-L., Perring, T. M., Krell, R. K., Farrar, C. A., and Gispert, C. 2006. Spatial distribution of Pierce's disease in the Coachella Valley: Implications for sampling. Am. J. Enol. Vitic. 57:220-225.

Park, Y.-L., Perring, T. M., Krell, R. K., Hashim-Buckey, J. M., and Hill, B. L. 2011. Spatial distribution of Pierce's disease related to incidence, vineyard characteristics, and surrounding land uses. Am. J. Enol. Vitic. 62:229-238.

Perring, T. M., Farrar, C. A., and Blua, M. J. 2001. Proximity to citrus influences Pierce's disease in Temecula Valley vineyards. Calif. Agric. 55:13-18.

Perring, T. M., Gruenhagen, N. M., and Farrar, C. A. 1999. Management of plant viral diseases through chemical control of insect vectors. Annu. Rev. Entomol. 44:457-481.

Pierce, N. B. 1892. The California Vine Disease. U. S. Dep. Agric. Div. Veg. Pathol. Bull. 2.

Pilkington, L. J., Irvin, N. A., Boyd, E. A., Hoddle, M. S., Triapitsyn, S. V., Carey, B. G., Jones, W. A., and Morgan, D. J. W. 2005. Introduced parasitic wasps could control glassy-winged sharpshooter. Calif. Agric. 59:223-228.

Pinheiro, J. C., and Bates, D. M. 2000. Mixed-Effects Models in S and S-PLUS. Springer, New York.
Purcell, A. H. 1974. Spatial patterns of Pierce's disease in the Napa Valley. Am. J. Enol. Vitic. 25:162-167.

Purcell, A. H. 1975. Role of the blue-green sharpshooter, Hordnia circellata, in the epidemiology of Pierce's disease of grapevines. Environ. Entomol. 4:745-752.

Purcell, A. H. 1977. Cold therapy of Pierce's disease of grapevines. Plant Dis. Rep. 61:514-518.

Purcell, A. H. 1997. Xylella fastidiosa, a regional problem or global threat? J. Plant Pathol. 79:99-105.

Purcell, A. H., and Finlay, A. H. 1979. Evidence for noncirculative transmission of Pierce's disease bacterium by sharpshooter leafhoppers. Phytopathology 69:393-395.

Rashed, A., Kwan, J., Baraff, B., Ling, D., Daugherty, M. P., Killiny, N., and Almeida, R. P. P. 2013. Relative susceptibility of Vitis vinifera cultivars to vector-borne Xylella fastidiosa through time. PLoS One 8:e55326.

Redak, R. A., Purcell, A. H., Lopes, J. R. S., Blua, M. J., Mizell, R. F., and Andersen, P. C. 2004. The biology of xylem fluid-feeding insect vectors of Xylella fastidiosa and their relation to disease epidemiology. Annu. Rev. Entomol. 49:243-270.

Saponari, M., Boscia, D., Nigro, F., and Martelli, G. P. 2013. Identification of DNA sequences related to Xylella fastidiosa in oleander, almond and olive trees exhibiting leaf scorch symptoms in Apulia (southern Italy). J. Plant Pathol. 95:668.

Severin, H. H. P. 1949. Transmission of the virus of Pierce's disease of grapevines by leafhoppers. Hilgardia 19:190-202.

Sicard, A., Zeilinger, A. R., Vanhove, M., Schartel, T. E., Beal, D. J., Daugherty, M. P., and Almeida, R. P. P. 2018. Xylella fastidiosa: Insights into an emerging plant pathogen. Annu. Rev. Phytopathol. 56:181-202.

Sisterson, M. S. 2008. Effects of insect-vector preference for healthy or infected plants on pathogen spread: Insights from a model. J. Econ. Entomol. 101:1-8.

Sisterson, M. S., and Stenger, D. C. 2013. Roguing with replacement in perennial crops: Conditions for successful disease management. Phytopathology 103:117-128

Sisterson, M. S., Yacoub, R., Montez, G., Grafton-Cardwell, E. E., and Groves, R. L. 2008. Distribution and management of citrus in California: Implications for management of glassy-winged sharpshooter. J. Econ. Entomol. 101:1041-1050

Sorensen, S. J., and Gill, R. J. 1996. A range extension of Homalodisca coagulata (Say) (Hemiptera: Clypeorrhyncha: Cicadellidae) to Southern California. Pan-Pac. Entomol. 72:160-161.

Wells, J. M., Raju, B. C., Hung, H.-Y., Weisburg, W. G., Mandelco-Paul, L., and Brenner, D. J. 1987. Xylella fastidiosa gen. nov. sp. Nov: Gramnegative, xylem-limited, fastidious plant bacteria related to Xanthomonas spp. Int. J. Syst. Bacteriol. 37:136-143.

Zeilinger, A. R., and Daugherty, M. P. 2014. Vector preference and host defense against infection interact to determine disease dynamics. Oikos 123: 613-622. 